\title{
Assessment of Volatile Sulfur Compounds in Adult and Pediatric Chronic Tonsillitis Patients Receiving Tonsillectomy
}

\author{
Kyu Young Choi ${ }^{1}$ Bum Sang Lee ${ }^{1}$ Jin Hwan $\mathrm{Kim}^{1} \cdot$ Jung Jun $\mathrm{Kim}^{2} \cdot$ Young Jang ${ }^{2}$ Jong Wook Choi' $\cdot$ Dong Jin Lee \\ ${ }^{1}$ Department of Otorhinolaryngology-Head and Neck Surgery, Hallym University Kangnam Sacred Heart Hospital, Seoul;
}

${ }^{2}$ Gwanak Ear Nose Throat Clinic, Seoul, Korea

Objectives. To study the volatile sulfur compound (VSC) concentration profile in chronic tonsillitis patients before and after tonsillectomy, and to evaluate the difference between adult and pediatric (children and adolescents) patients.

Methods. Thirty adult patients (older than 20 years old) and 30 pediatric patients (younger than 20 years old) who were assigned to get tonsillectomy due to chronic tonsillitis were enrolled in this prospective nonrandomized clinical study. The concentrations of the three main VSCs related to halitosis (hydrogen sulfide, methyl mercaptan, and dimethyl sulfide) were assessed in each patient using a portable chromatograph (Oral Chroma ${ }^{\mathrm{TM}}$ ) at 1 day before operation, postoperative 1 day, 1 week, and 2 weeks.

Results. Average concentration of hydrogen sulfide, methyl mercaptan, and dimethyl sulfide preoperatively were $99.5 \mathrm{ppb}$, $24.6 \mathrm{ppb}$, and $9.45 \mathrm{ppb}$ in adult patients, and $97.4 \mathrm{ppb}, 26 \mathrm{ppb}$, and $10.5 \mathrm{ppb}$ in pediatric patients, respectively. The concentrations of the three VSCs in both groups were highest in first day after surgery, and decreased signigicantly after 2 weeks compared to preoperative values $(P<0.001)$. There was no significant difference of the concentration of the three VSCs between adult and pediatric patients in any time point.

Conclusion. The concentrations of hydrogen sulfide, methyl mercaptan, and dimethyl sulfide decreased significantly after tonsillectomy in chronic tonsillitis patients. The concentrations of the three VSCs were not significantly different between pediatric and adult patients before and after tonsillectomy.

Keywords. Tonsillitis; Tonsillectomy; Halitosis; Sulfur Compounds; Chromatography; Gas

\section{INTRODUCTION}

Halitosis is defined as a bad or unpleasant odor emanating from the mouth. Various causes of halitosis have been reported; however, chronic tonsillitis is one of the most common otolaryngologic causes of halitosis in adult patients. Frequent inflammation of the tonsil can result in changes on the surface of tonsillar crypts and form tonsillolithiasis (tonsillar calculi), which subse-

- Received August 26, 2017

Revised December 22, 2017

Accepted January 30, 2018

- Corresponding author: Dong Jin Lee

Department of Otorhinolaryngology-Head and Neck Surgery, Hallym

University Kangnam Sacred Heart Hospital, 1 Singil-ro, Yeongdeungpo-gu, Seoul 07441, Korea

Tel: +82-2-829-5632, Fax: +82-2-842-5217, E-mail: djlee@hallym.or.kr quently causes malodor. The changes of the constituent of normal flora by chronic tonsillitis also contribute to halitosis. However, limited data are available on tonsillar halitosis because most of halitosis cases are due to intraoral causes and managed usually by dentists. Since halitosis is not a usual complaint in pediatric patients, most halitosis studies have been performed in adult patients.

Oral Chroma ${ }^{\mathrm{TM}}$ is a portable halitosis measuring device. It can classify the volatile sulfur compounds (VSCs), major causative factor in halitosis, into the three major components of hydrogen sulfide $\left(\mathrm{H}_{2} \mathrm{~S}\right)$, methyl mercaptan $\left(\mathrm{CH}_{3} \mathrm{SH}\right)$, and dimethyl sulfide $\left(\mathrm{CH}_{3} \mathrm{SCH}_{3}\right)$, and measure the concentration of each gas. Measuring individualVSC components helps to identify the cause of halitosis, and to check the effect of treatment.

The concentrations of the three VSCs contributing to halitosis

Copyright (C) 2018 by Korean Society of Otorhinolaryngology-Head and Neck Surgery.

This is an open-access article distributed under the terms of the Creative Commons Attribution Non-Commercial License (http://creativecommons.org/licenses/by-nc/4.0)

which permits unrestricted non-commercial use, distribution, and reproduction in any medium, provided the original work is properly cited. 
in adult and pediatric tonsillitis patients have never been reported in the literature. Moreover, the changes of the concentration of theVSCs over time after tonsillectomy, and the difference between adult and pediatric patients have never been studied. The aim of this study was to evaluate the concentration profile of the three major VSCs in chronic tonsillitis patients (with or without halitosis) and to compare them between adult and pediatric (children and adolescents) patients by use of Oral Chroma ${ }^{\mathrm{TM}}$, in patients who underwent tonsillectomy.

\section{MATERIALS AND METHODS}

From September 2016 to April 2017, 30 pediatric patients (younger than 20 years of age) and 30 adult patients (older than 20 years of age) with recurrent tonsillitis who were assigned to receive tonsillectomy and/or adenoidectomy, were prospectively recruited from Hallym University Kangnam Sacred Heart Hospital in Korea. Exclusion criteria were patients with previous operation, bleeding disorder, medical diseases, metabolic syndrome, psychological problem, craniofacial anomaly, peritonsillar abscess or neoplasm. Smokers, heavy alcoholic drinkers (more than $30 \mathrm{~g}$ alcohol/day), drug users, and those with dental diseases, sinusitis, laryngopharyngeal reflux disease, or thick tongue coat were also excluded in order to avoid other causes that can affect the production of VSCs in the oral cavity. General characteristics of the patients were identified, as well as the presence of halitosis were asked to each patient.

VSCs of each patient were measured by a portable gas chromatograph (Oral Chroma ${ }^{\mathrm{TM}}$, Abimedical; Abilit Corp., Osaka, Japan) according to the manufacturers' instructions. All participants were advised not to use mouthwash, toothpaste, chewing gum, spicy or seasoned food for at least 3 hours and not to fast for more than 4 hours before the tests. A disposable $1 \mathrm{~mL}$ syringe was inserted into the oral cavity, and the patients were asked to nose breathe while keeping the oral cavity sealed and unventilated for 1 minute. Then, $1.0 \mathrm{~mL}$ of the air sample was drawn by the syringe, and after expelling out $0.5 \mathrm{~mL}$ of the collected sample to eliminate unwanted air, the remaining $0.5 \mathrm{~mL}$ was injected into the Oral Chroma ${ }^{\mathrm{TM}}$. The concentrations of $\mathrm{H}_{2} \mathrm{~S}$,

\section{H I G H L I G G H T T S}

- Volatile sulfur compounds (VSCs) were analyzed in chronic tonsillitis patients.

- The concentrations of VSCs decreased significantly after tonsillectomy.

- There were no significant differences in the VSCs between adult and pediatric patients.

- The VSCs related to halitosis must not be underestimated in pediatric tonsillitis.
$\mathrm{CH}_{3} \mathrm{SH}, \mathrm{CH}_{3} \mathrm{SCH}_{3}$ were automatically calculated and recorded. The VSCs were examined in the afternoon four times totally for each patient, 1 day ahead of tonsillectomy, postoperative 1 day, postoperative 1 week, and postoperative 2 weeks.

The surgeries were performed under general anesthesia with the patient in Rose position. Tonsillectomy was performed by using monopolar electrocautery (Electrosurgical Pencil, Valleylab; Medtronic, Boulder, CO, USA) in the conventional extracapsular dissection method. After tonsillectomies, adenoidectomy was done (if needed) by using microdebrider (Straightshot M4 Microdebrider; Medtronic, Minneapolis, MN, USA) for tissue removal and monopolar suction cautery (Valleylab Suction Coagulator, Medtronic) for bleeding control. All surgeries were performed by one experienced otolaryngologist (DJL). Postoperative medications for 10 days were same for all patients; paracetamol or ibuprofen or both, and amoxicillin in doses appropriate to the weight of the patient. Oral gargle was recommended with water. All patients were discharged home the next day after surgery and then followed-up in the outpatient clinic on the 7 th and 14 th postoperative day.

The prospective nonrandomized clinical study was conducted according to the principles expressed in the Declaration of Helsinki, and was approved by the Institutional Review Board of Hallym University Kangnam Sacred Heart Hospital (IRB No. 2016-10-70). Written informed consent was obtained from each patient or the caregiver. Statistical analysis was performed using IBM SPSS ver. 20.0 (IBM Corp., Armonk, NY, USA). The chisquare test, Fisher exact test, or linear by linear association were used to examine differences in sex and clinical characteristics between the adult and the pediatric patient group. Paired $t$-test was used to compare the concentration of VSCs between the two groups, and between pre- and postoperative time point. $P$ values $<0.05$ were considered statistically significant.

\section{RESULTS}

A total of 60 patients who agreed to participate in the trial were initially enrolled. However, one child patient who did not show up at the postoperative period and another adult patient who had underwent a second operation for bleeding control were excluded. By including new two patients, 30 pediatric patients and 30 adult patients were finally enrolled in this study. Two more patients had mentioned episode of postoperative bleeding; however, the bleeding stopped with observation in all cases which did not influence the postoperative analysis of VSCs. No other serious complication was noted after the surgeries. The postoperative histopathological study reported chronic tonsillitis in all patients.

The clinical characteristics of the participants are presented in Table 1 . The mean age of the pediatric patients was $14 \pm 2$ years (range, 7 to 19 years), and the mean age of the adult patients 
was $29 \pm 4$ years (range, 20 to 51 years). There were 17 male and 13 female patients in the pediatric group, and 20 male and 10 female patients in the adult group. Sixteen pediatric patients had undergone adenoidectomy simultaneously, while all the adult patients had got only tonsillectomy. No patients in the pediatric group had complained of halitosis before the operation. However, the number of patients who had noted halitosis preoperatively was eight $(26.7 \%)$ in the adult group. Four patients in the adult group had tonsilloliths, and five patients had history of peritonsillar abscess. The sex distribution was not significantly different between the pediatric group and the adult group, however, the intraoperative findings of the tonsils showed difference between the two groups (Table 1).

The mean concentrations of VSCs for each group according to time period are provided in Table 2. Preoperatively, the mean values of $\mathrm{H}_{2} \mathrm{~S}$ were lower in the pediatric group, while the mean

Table 1. Clinical characteristics of the patients

\begin{tabular}{lccc}
\hline Characteristic & Pediatric $(\mathrm{n}=30)$ & Adult $(\mathrm{n}=30)$ & P-value \\
\hline Mean age $(\mathrm{yr})$ & 14 & 29 & - \\
Sex & & & 0.596 \\
Male:female & $17(57): 13(43)$ & $20(67): 10(33)$ & \\
Halitosis & 0 & $8(26.7)$ & 0.005 \\
Tonsil size & & & $<0.001$ \\
Grade 1 & $1(3)$ & $3(10)$ & \\
Grade 2 & $4(13)$ & $17(57)$ & \\
Grade 3 & $10(33)$ & $8(27)$ & \\
Grade 4 & $15(50)$ & $2(7)$ & \\
Tonsil adhesion state & & & \\
Mild & $21(70)$ & $7(23)$ & \\
Moderate & $8(27)$ & $11(37)$ & \\
Severe & $1(3)$ & $12(40)$ & \\
\hline
\end{tabular}

Values are presented as number (\%). concentrations of $\mathrm{CH}_{3} \mathrm{SH}$ and $\mathrm{CH}_{3} \mathrm{SCH}_{3}$ were slightly higher in the pediatric group. However, the values were not much different between the two groups and no significant difference were found $(P>0.05)$. The mean concentrations of the three VSCs were increased right after surgery, however, they tend to descend continuously over time, highest at postoperative day 1 and lowest at postoperative week 2 (Fig. 1). Compared to the preoperative values, the concentrations of the three VSCs at postoperative week 2 were significantly lower in both groups $(P<0.001)$ (Fig. 1). All the eight patients who had halitosis preoperatively revealed improvement of halitosis at postoperative

Table 2. Mean concentration of $\mathrm{H}_{2} \mathrm{~S}, \mathrm{CH}_{3} \mathrm{SH}$, and $\mathrm{CH}_{3} \mathrm{SCH}_{3}$ according to the time period

\begin{tabular}{|c|c|c|c|}
\hline Concentration & Pediatric $(n=30)$ & Adult $(n=30)$ & $P$-value \\
\hline \multicolumn{4}{|c|}{ Preoperative (ppb) } \\
\hline $\mathrm{H}_{2} \mathrm{~S}$ & $97.4 \pm 32.1$ & $99.5 \pm 49.5$ & 0.846 \\
\hline $\mathrm{CH}_{3} \mathrm{SH}$ & $26.0 \pm 8.7$ & $24.6 \pm 12.1$ & 0.617 \\
\hline $\mathrm{CH}_{3} \mathrm{SCH}_{3}$ & $10.5 \pm 5.9$ & $9.4 \pm 7.3$ & 0.499 \\
\hline \multicolumn{4}{|c|}{ Postoperative day 1 (ppb) } \\
\hline $\mathrm{H}_{2} \mathrm{~S}$ & $138.1 \pm 36.5$ & $143.3 \pm 55.3$ & 0.671 \\
\hline $\mathrm{CH}_{3} \mathrm{SH}$ & $40.5 \pm 10.5$ & $36.4 \pm 14.1$ & 0.211 \\
\hline $\mathrm{CH}_{3} \mathrm{SCH}_{3}$ & $15.8 \pm 6.7$ & $15.6 \pm 10.0$ & 0.952 \\
\hline \multicolumn{4}{|c|}{ Postoperative wk 1 (ppb) } \\
\hline $\mathrm{H}_{2} \mathrm{~S}$ & $126.3 \pm 35.0$ & $132.7 \pm 55.3$ & 0.596 \\
\hline $\mathrm{CH}_{3} \mathrm{SH}$ & $32.8 \pm 10.1$ & $27.6 \pm 13.5$ & 0.098 \\
\hline $\mathrm{CH}_{3} \mathrm{SCH}_{3}$ & $12.5 \pm 6.2$ & $11.0 \pm 8.8$ & 0.447 \\
\hline \multicolumn{4}{|c|}{ Postoperative wk 2 (ppb) } \\
\hline $\mathrm{H}_{2} \mathrm{~S}$ & $71.5 \pm 27.4$ & $71.6 \pm 30.1$ & 0.989 \\
\hline $\mathrm{CH}_{3} \mathrm{SH}$ & $15.1 \pm 7.8$ & $14.4 \pm 7.1$ & 0.730 \\
\hline $\mathrm{CH}_{3} \mathrm{SCH}_{3}$ & $3.7 \pm 2.8$ & $3.1 \pm 2.8$ & 0.358 \\
\hline
\end{tabular}

Values are presented as mean \pm standard deviation.

$\mathrm{H}_{2} \mathrm{~S}$, hydrogen sulfide; $\mathrm{CH}_{3} \mathrm{SH}$, methyl mercaptan; $\mathrm{CH}_{3} \mathrm{SCH}_{3}$, dimethyl sulfide.
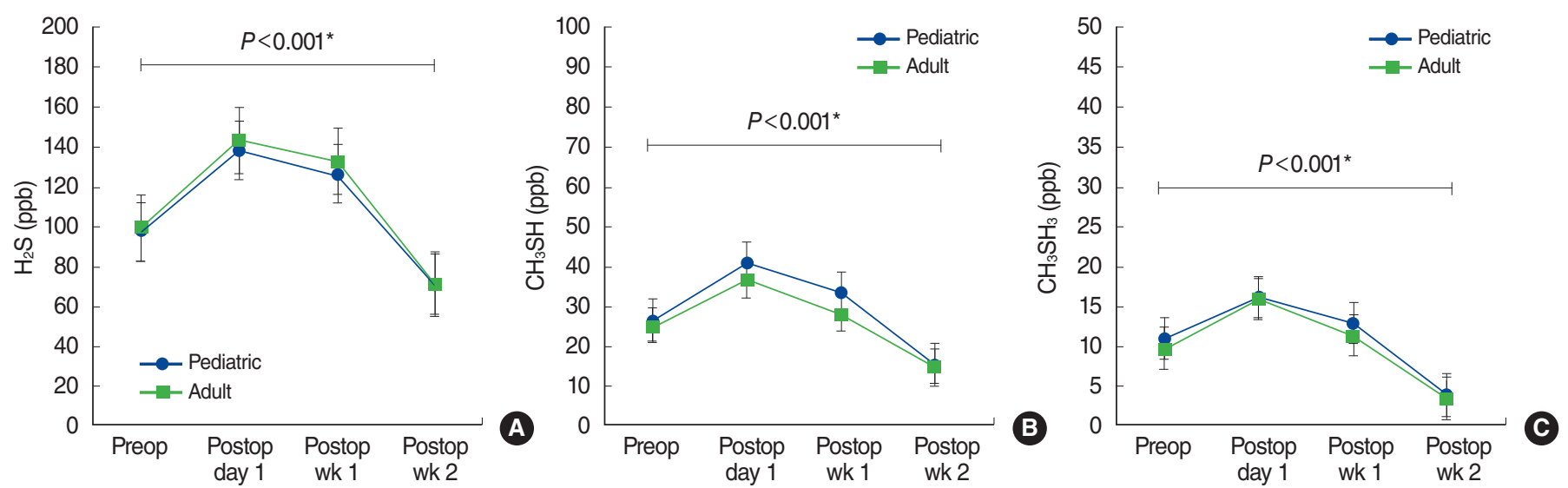

Fig. 1. The mean concentrations of $(A)$ hydrogen sulfide $\left(\mathrm{H}_{2} \mathrm{~S}\right)$, (B) methyl mercaptan $\left(\mathrm{CH}_{3} \mathrm{SH}\right)$, and $(\mathrm{C})$ dimethyl sulfide $\left(\mathrm{CH}_{3} \mathrm{SCH} \mathrm{H}_{3}\right)$ according to the time point in pediatric and adult chronic tonsillitis patients. The mean values of the three volatile sulfur compounds continued to decrease over time after tonsillectomy, and significant difference were noted at postoperative (Postop) week 2 compared to preoperative (Preop) period in both pediatric and adult groups. However, the concentrations of the three volatile sulfur compounds were not significantly different between the pediatric group and the adult group at any time point. *Statistically significant. 
week 2. There were no significant differences of the mean concentrations of three VSCs between the pediatric group and the adult group, at any postoperative time point $(P>0.05)$ (Table 2$)$.

\section{DISCUSSION}

Halitosis is a common disorder with a prevalence of $10 \%-30 \%$ within the general population [1]. It can lead to a very unpleasant symptom, as well as social restriction and decreased quality of life. The diverse cause of halitosis can be divided into intraoral causes, extraoral causes, psychologic factors, and transient factors (such as food ingestion, alcohol, smoking, etc.). Intraoral halitosis, which is the most common cause affecting $80 \%-90 \%$ of all cases [2], mainly results from the byproducts of bacterial metabolic degradation of organic substrates in the oral cavity [3]. Intraoral conditions that contributes to this process include poor oral hygiene, tongue coating, mucosal ulcerations, impacted food or debris, dental problems such as gingivitis and periodontitis, etc. [1]. The microbial proteolysis of proteins to peptides, amino acids, and substrates with free thiol groups, gives rise to VSCs which are the major contributors to halitosis $[3,4]$. The three most important VSCs in the development of oral malodor are $\mathrm{H}_{2} \mathrm{~S}, \mathrm{CH}_{3} \mathrm{SH}$, and $\mathrm{CH}_{3} \mathrm{SCH}_{3}$, accounting for roughly $90 \%$ $[5,6]$. $\mathrm{CH}_{3} \mathrm{SH}$ and to a lesser extent $\mathrm{H}_{2} \mathrm{~S}$ are mainly detected in intraoral halitosis, with $\mathrm{H}_{2} \mathrm{~S}$ being the major component in periodontal disease, while $\mathrm{CH}_{3} \mathrm{SCH}_{3}$ is more associated with extraoral (blood-borne) halitosis [7-9].

Extraoral halitosis accounts for $10 \%-20 \%$ of bad breath. They are mainly related to otolaryngology and respiratory diseases, while gastrointestinal, liver/renal diseases, and other metabolic syndromes are minor causes [10]. Previous reports have suggested that the most common cause of extraoral halitosis is the tonsils [11,12], and chronic tonsillitis is the actual cause of idiopathic halitosis in most cases [13]. It has also been reported that about $3 \%$ of all halitosis are secondary to pathology of the palatine tonsils [14]. The palatine tonsils continuously sample antigens from inhaled air, foods, and the microbiota. The branching and interconnecting crypt system makes anaerobic bacteria to reside, which makes palatine tonsil the most suitable site for their activity in the upper respiratory tract $[13,15]$. Continuing immunologic processes and inflammation are usually present in the palatine tonsils that increase the production of VSCs.

The exact causative odorous volatile compound responsible in tonsillitis has not been identified [16]; however, it can be similar to those in intraoral halitosis because the oropharynx area is adjacent to the oral cavity and the VSC-forming anaerobic oral bacteria also constitute the oropharyngeal microbiota [17]. As often as not, tonsil stone (tonsillolith) can be formed in the tonsillar crypts from the retained exfoliated epithelium cells, keratin debris, and foreign particles. Anaerobic bacteria detected in tonsilloliths are Eubacterium, Fusobacterium, Porphyromonas,
Prevotella, Selenomonas, and Tanerella species, all of which are associated with VSCs production [18]. It has been reported that approximately $77 \%$ of patients with chronic caseous tonsillitis have intermittent halitosis [14,15], and that tonsilloliths give tenfold increased risk for elevated concentration of VSCs [10]. Nevertheless, limited studies have been reported aboutVSCs related to tonsils, compared to a large number of researches concerning VSCs in intraoral halitosis.

Methods in analyzing oral malodor include organoleptic measurement, sulphide monitoring, and gas chromatography, etc. Organoleptic measurement, clinician smelling and grading the patient's breath odor, is widely used clinically but is a subjective test. Halimeter, a portable sulfide monitor, is an objective test; however, it cannot differentiate between the three VSCs. It is sensitive to $\mathrm{H}_{2} \mathrm{~S}$, but less sensitive to $\mathrm{CH}_{3} \mathrm{SH}$ and almost insensitive to $\mathrm{CH}_{3} \mathrm{SCH}_{3}$ [19]. Gas chromatography can specifically determine the concentrations of the VSCs by using a sulfur detector. It is an objective, reproducible, and reliable apparatus, and is considered as the gold standard of halitosis measurement [13, 20]. However, it is not easily implemented in clinical settings due to its relatively high cost and the requirement of laboratory and trained personnel. In this sense, Oral Chroma ${ }^{\mathrm{TM}}$ which is a portable gas chromatograph, has been produced to easily detect VSCs in clinical settings. It is inexpensive and can be used in outpatient clinic. Oral Chroma ${ }^{\mathrm{TM}}$ measures the main three VSCs associated with halitosis with equally high sensitivities [3], which can detect both intra- as well as extraoral halitosis and differentiate them $[2,21]$. Tangerman and Winkel [16] have suggested the threshold of concentration to determine contributor of halitosis of $\mathrm{H}_{2} \mathrm{~S}, \mathrm{CH}_{3} \mathrm{SH}$, and $\mathrm{CH}_{3} \mathrm{SCH}_{3}$ as $95 \mathrm{ppb}, 12 \mathrm{ppb}$, and $24 \mathrm{ppb}$, respectively.

In this study, the concentrations of the major three VSCs in tonsillitis patients were identified by using Oral $\mathrm{Chroma}^{\mathrm{TM}}$. The VSCs identified in our study were considered to be related with tonsillitis by our exclusion criteria and the management of intraoral conditions before checking with Oral Chroma ${ }^{\mathrm{TM}}$. Also, the fact that the concentrations decreased after tonsillectomy implies that the VSCs measured in our study were related to the tonsils. The concentrations of the VSCs in chronic tonsillitis patients were not significantly different between adult and pediatric patients in both pre- and postoperative periods. The pediatric patients with tonsillitis, although none of them complained of halitosis, revealed similar concentrations of VSCs compared to adult patients even before treatment.

The treatment of halitosis should be targeted to the causal factor, so identifying the cause of halitosis in the individual patient is most important. However, confirmation of the origin of halitosis is not easy in practice, and much effort including thorough physical examination are needed. Management of intraoral halitosis includes mechanical and chemical removal of bacterial and organic substrates in the oral cavity, treating dental problems, and improving oral hygiene, etc. For tonsillar halito- 
sis, medical treatment, radiofrequency ablation, laser or coblation cryptolysis, and tonsillectomy can be performed [22]. Tonsillectomies are generally performed for halitosis when treatment with topical antiseptics and oral antibiotics does not bring relief [10], or when the tonsillar crypts are found to contain malodorous substrates [14]. However, the effect of tonsillectomy on halitosis has not been clarified. Al-Abbasi [23] reported that tonsillectomy in 44 chronic tonsillitis patients resulted in complete remission of halitosis in $79.5 \%$ patients, and incomplete remission in $20.4 \%$; however, the tonsil smelling test was negative in all subjects at 8 weeks postoperatively. Our study also showed significant decrease of all three major VSCs on chronic tonsillitis patients at 2 weeks after tonsillectomy when other intraoral causes of halitosis are controlled. It can be suggested from our study that tonsillectomy lowers the production of VSCs, and that it may contribute to the treatment of halitosis in both adult and pediatric tonsillitis patients.

Halitosis in children are often underestimated, because most of the halitosis patients are adults and children do not usually complain of halitosis. Halitosis and the concentrations of VSCs in pediatric tonsillitis patients have not been studied so far. However, pediatric halitosis necessitates a value of evaluation since age have been reported to have no relationship with halitosis $[10,24]$, and almost $40 \%$ of children participated in the study of Villa et al. [20] had halitosis. In literature, the halitosis of tonsillitis patients have never been compared between children and adults. This is the first study to investigate the VSCs of child tonsillitis patients before and after tonsillectomy. Our study shows that halitosis in pediatric tonsillitis patients need close attention because the values of VSCs both before and after surgery were similar to those of the adult patients.

The limitations of our study are that the evaluation of VSCs might be influenced by other causes of oral malodor (VSCs produced by intraoral factors may have been included in the results). Although maximum efforts had been made to exclude the other factors in this study, a more standardized method to measure only the tonsillar VSCs and subsequently avoid bias need to be established in the future. Secondly, we only checked the concentrations of the majorVSCs, and other compounds related to halitosis such as volatile short-chain fatty acids, polyamines, phenyl compounds, alkanes, ketones, nitrogen-containing compounds, etc. were not considered in this study. Also, control group who did not check the VSCs during tonsillectomy had not been included. To accurately diagnose halitosis and to be more helpful in clinical practice, additional evaluation on organoleptic assessment and subjective patient symptoms of bad breath are needed. And lastly, the effect of tonsillectomy on halitosis was investigated for only short time, and the sample size was relatively small. Although we had enrolled 30 patients in each group to fulfill sufficiently large sample size according to central limit theorem, further long-term clinical trials with larger sample size are required to confirm these results and to better understand the relationship between halitosis and tonsillectomy.

In conclusion, the major VSCs contributing to halitosis in chronic tonsillitis patients were evaluated and compared before and after tonsillectomy, that were also compared between adult and pediatric patients. The three VSCs were significantly decreased after tonsillectomy in both adult and pediatric patients. However, even though the presence of the symptom of halitosis in pediatric patients was significantly lower compared to adult patients, the concentrations of the VSCs showed no significant age-specific differences. In clinical practice, halitosis due to chronic tonsillitis can be managed successfully with tonsillectomy, and the VSCs related to halitosis can also be considered similarly in pediatric patients as in adult patients.

\section{CONFLICT OF INTEREST}

No potential conflict of interest relevant to this article was reported.

\section{REFERENCES}

1. Meskin LH.A breath of fresh air. J Am Dent Assoc. 1996 Sep;127(9): 1282-4.

2. Sinjari B, Murmura G, Caputi S, Ricci L, Varvara G, Scarano A. Use of Oral ChromaTM in the assessment of volatile sulfur compounds in patients with fixed protheses. Int J Immunopathol Pharmacol. 2013 Jul-Sep;26(3):691-7.

3. Salako NO, Philip L. Comparison of the use of the Halimeter and the Oral ChromaTM in the assessment of the ability of common cultivable oral anaerobic bacteria to produce malodorous volatile sulfur compounds from cysteine and methionine. Med Princ Pract. 2011;20(1):75-9.

4. Ayers KM, Colquhoun AN. Halitosis: causes, diagnosis, and treatment. N Z Dent J. 1998 Dec;94(418):156-60.

5. Takahashi N. Microbial ecosystem in the oral cavity: metabolic diversity in an ecological niche and its relationship with oral diseases. International Congress Series. 2005 Sep;1284:103-12.

6. Porter SR, Scully C. Oral malodour (halitosis). BMJ. 2006 Sep;333 (7569):632-5.

7. Nakano Y, Yoshimura M, Koga T. Methyl mercaptan production by periodontal bacteria. Int Dent J. 2002 Jun;52 Suppl 3:217-20.

8. Suarez FL, Furne JK, Springfield J, Levitt MD. Morning breath odor: influence of treatments on sulfur gases. J Dent Res. 2000 Oct;79 (10):1773-7.

9. Tangerman A, Winkel EG. Intra- and extra-oral halitosis: finding of a new form of extra-oral blood-borne halitosis caused by dimethyl sulphide. J Clin Periodontol. 2007 Sep;34(9):748-55.

10. Rio AC, Franchi-Teixeira AR, Nicola EM. Relationship between the presence of tonsilloliths and halitosis in patients with chronic caseous tonsillitis. Br Dent J. 2008 Jan;204(2):E4.

11. Filippi A, Muller N. Real and psychological halitosis: findings, diagnoses and outcomes of a halitosis clinic. Schweiz Monatsschr Zahnmed. 2006;116(2):129-35.

12. Tulupov DA, Bakhmutov DN, Karpova EP. Halitosis concomitant with chronic ENT pathology in children. Vestn Otorinolaringol. 2013;(5):59-61. 
13. Finkelstein Y,Talmi YP, Ophir D, Berger G. Laser cryptolysis for the treatment of halitosis. Otolaryngol Head Neck Surg. 2004 Oct;131 (4):372-7.

14. Ferguson M,Aydin M, Mickel J. Halitosis and the tonsils: a review of management. Otolaryngol Head Neck Surg. 2014 Oct;151(4):56774.

15. Dal Rio AC, Passos CA, Nicola JH, Nicola EM. CO2 laser cryptolysis by coagulation for the treatment of halitosis. Photomed Laser Surg. 2006 Oct;24(5):630-6.

16. Tangerman A, Winkel EG. Extra-oral halitosis: an overview. J Breath Res. 2010 Mar;4(1):017003.

17. Iwamura Y, Hayashi J, Sato T, Sato S, Murakami T, Fujimura T, et al. Assessment of oral malodor and tonsillar microbiota after gargling with benzethonium chloride. J Oral Sci. 2016 Mar;58(1):83-91.

18. Tsuneishi M, Yamamoto T, Kokeguchi S, Tamaki N, Fukui K, Watanabe T. Composition of the bacterial flora in tonsilloliths. Microbes Infect. 2006 Aug;8(9-10):2384-9.
19. Furne J, Majerus G, Lenton P, Springfield J, Levitt DG, Levitt MD. Comparison of volatile sulfur compound concentrations measured with a sulfide detector vs. gas chromatography. J Dent Res. 2002 Feb;81(2):140-3.

20.Villa A, Zollanvari A, Alterovitz G, Cagetti MG, Strohmenger L,Abati S. Prevalence of halitosis in children considering oral hygiene, gender and age. Int J Dent Hyg. 2014 Aug;12(3):208-12.

21. van den Broek AM, Feenstra L, de Baat C. A review of the current literature on aetiology and measurement methods of halitosis. J Dent. 2007 Aug;35(8):627-35.

22. Harvey-Woodworth CN. Dimethylsulphidemia: the significance of dimethyl sulphide in extra-oral, blood borne halitosis. Br Dent J. 2013 Apr;214(7):E20.

23. Al-Abbasi AM. Tonsillectomy for the treatment of halitosis. Niger $\mathrm{J}$ Med. 2009 Jul-Sep;18(3):295-8.

24. Gokdogan O, Catli T, Ileri F. Halitosis in otorhinolaryngology practice. Iran J Otorhinolaryngol. 2015 Mar;27(79):145-53. 